Document downloaded from:

http://hdl.handle.net/10251/37327

This paper must be cited as:

Conejero Casares, JA.; Martínez Jiménez, F. (2011). Chaotic differential operators. Revista- Real Academia de Ciencias Exactas Fisicas Y Naturales Serie a Matematicas. 105(2):423-431. doi:10.1007/s13398-011-0026-6.

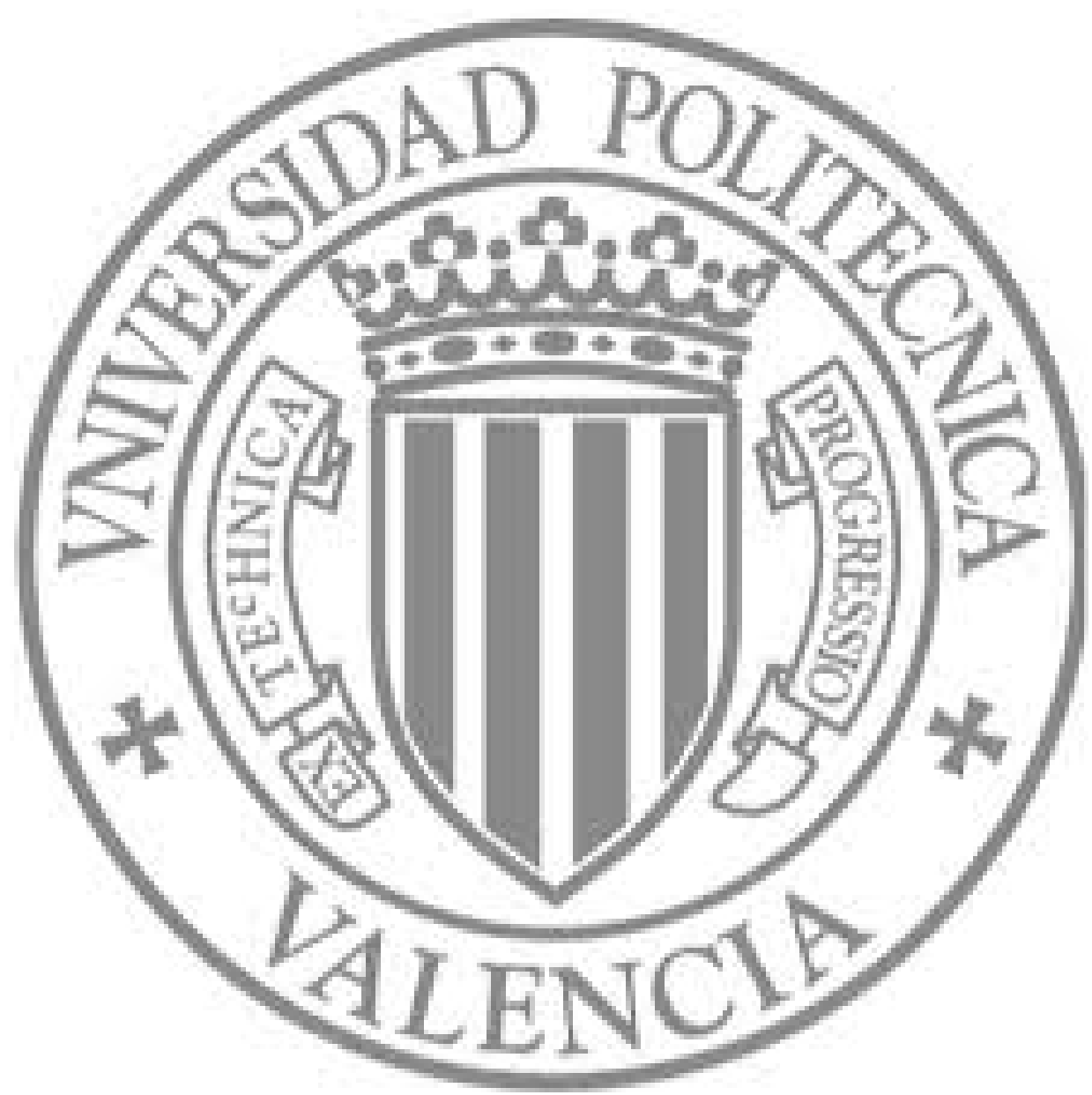

The final publication is available at

http://link.springer.com/article/10.1007\%2Fs13398-011-0026-6

Copyright Springer Verlag (Germany) 


\title{
Chaotic Differential Operators
}

\author{
J.A. Conejero · F. Martínez-Giménez
}

Received: date / Accepted: date

Abstract We give sufficient conditions for chaos of (differential) operators on Hilbert spaces of entire functions. To this aim we establish conditions on the coefficients of a polynomial $P(z)$ such that $P(B)$ is chaotic on the space $\ell^{p}$, where $B$ is the backward shift operator.

Keywords Chaotic operators, hypercyclic operators, differential operators, backward shifts

\section{Mathematics Subject Classification (2000) 47A16}

According to Devaney [7], a continuous map on a metric space is chaotic provided it is topologically transitive and it has a dense set of periodic points. This paper deals with the chaotic behaviour of a class of bounded linear maps (operators) defined on separable Banach spaces. Within this context, it is well known that topological transitivity is equivalent to the existence of a dense orbit and this property is known for operators as hypercyclicity. Therefore we have that operators defined on separable Banach spaces are chaotic if and only if they are hypercyclic and admit a dense set of periodic points. We refer to the recent monographs $[1$, 10] for good introductions to this subject.

The first source of motivation for this work is a nice result of Godefroy and Shapiro [8] stating that any differential operator $P(D)$ not a multiple of the identity is chaotic in the space of entire functions $\mathcal{H}(\mathbb{C})$, endowed with its natural compactopen topology. As it was pointed out by them, it is clear that a similar result cannot be stated for bounded differential operators on Banach spaces since a suitable small multiple of a bounded operator is a contraction, and cannot be hypercyclic and hence not chaotic. From this point of view it is interesting to give conditions under which a differential operator $P(D)$ defined on a Banach space of entire functions is chaotic. In the same direction Shapiro [19] proved that $G$ is a simply connected domain if and only if $P(D)$ is chaotic on $\mathcal{H}(G)$ for any non-constant polynomial $P(z)$.

J. Alberto Conejero · Félix Martínez-Giménez

IUMPA \& Departamento Matemática Aplicada

Universidad Politécnica de Valencia

46022 Valencia, Spain

E-mail: aconejero@mat.upv.es; fmartinez@mat.upv.es 
The second source of motivation is related with one of the first examples of hypercyclic operators. Rolewicz [17] proved that multiples $\lambda B$ of the backward shift operator $B\left(x_{1}, x_{2}, \ldots\right):=\left(x_{2}, x_{3}, \ldots\right)$ are hypercyclic on the space $\ell^{1}$ of absolutely summable sequences if and only if $|\lambda|>1$; in fact, they are chaotic. Since then, (weighted) shift operators defined on sequence spaces have become a usual ground to study linear dynamics: Hypercyclicity for weighted backward shifts defined on $\ell^{p}$ was characterized by Salas and he also showed that any perturbation of the identity by a weighted backward shift is always hypercyclic [18]. Following this direction, characterizations for hypercyclic and chaotic weighted backward shifts defined on general sequence spaces were obtained in $[9,13]$, and characterizations for chaotic perturbations of the identity by weighted backward shifts are also in [13]. Other results for the linear dynamics of operators of the form $P(T)$, where $T$ is an operator and $P(z)$ is a polynomial or a more general function, can be found in $[2,5,6,11,14,15]$.

Finally, we would like to mention that the first step to get conditions for chaotic differential operators defined on Banach spaces of entire functions is to study chaos for operators $P(B)$, where $P(z)$ is a complex polynomial and $B$ is the backward shift operator. This is a consequence of topological conjugacy when representing the space of functions as a weighted $\ell^{p}$-space and the operator of differentiation as a backward shift (see examples in [14, Section 4]).

For strictly positive weight sequences $v:=\left(v_{n}\right)_{n}$, let

$$
\ell^{p}(v):=\left\{\left(x_{n}\right)_{n} \in \mathbb{C}^{\mathbb{N}},\|x\|^{p}:=\sum_{n=1}^{\infty}\left|x_{n}\right|^{p} v_{n}<\infty\right\}, 1 \leq p<\infty
$$

be the associated weighted $\ell^{p}$-space. It is easy to check that the weight condition

$$
\sup _{i} \frac{v_{i}}{v_{i+1}}<\infty
$$

characterizes when $B$ is a bounded operator on $\ell^{p}(v)$. This condition will always be assumed to hold. If the weight sequence $v$ coincides with the sequence of ones, the corresponding space will be denoted as $\ell^{p}$.

Our aim is to state the chaotic behaviour of $P(B)$ on $\ell^{p}(v)$, where $P(z)$ is a non-constant complex polynomial. We will start with the "unweighted" case, this is, $v$ is the sequence of ones. To get chaos for $P(B)$ on $\ell^{p}$ we will use the well known Eigenvalue Criterion for Chaos (see any of the monographs $[1,10]$ or $[8$, $3,6,14]$ for examples using it). In this framework, having in mind that the point spectrum $\sigma_{p}(B)=\mathbb{D}$ (the complex unit disc, its closure and boundary will be denoted as $\mathbb{D}, \overline{\mathbb{D}}$ and $\mathbb{T}$ respectively), and also that $P(\lambda)$ is an eigenvalue of $P(B)$ if $\lambda$ is an eigenvalue of $B$, this Criterion reads as $P(B)$ is chaotic on $\ell^{p}$ if and only if $P(\mathbb{D}) \cap \mathbb{T} \neq \emptyset$ (see [8]). Even more, since $P(\mathbb{D})$ is open and connected it suffices to show that $P(\mathbb{D})$ intersects $\mathbb{D}$ and $\mathbb{C} \backslash \overline{\mathbb{D}}$. At this point we would like to remark that to find a full geometrical description of $P(\mathbb{D})$ may be very hard in general and also that we want computable conditions for chaos, therefore our results should involve only the coefficients of $P(z)$. In the pursuit of this, the next three lemmas provide conditions on the coefficients of $P(z)$ such that $P(\mathbb{D})$ intersects $\mathbb{D}$ and $\mathbb{C} \backslash \overline{\mathbb{D}}$. 
Lemma 1 For any complex polynomial $P(z)=p_{0}+p_{1} z+\ldots+p_{n} z^{n}$ we have

$$
\left(\sum_{i=0}^{n}\left|p_{i}\right|^{2}\right)^{1 / 2} \leq \sup _{|z| \leq 1}|P(z)| \leq \sum_{i=0}^{n}\left|p_{i}\right|
$$

In particular, if $\sum_{i=0}^{n}\left|p_{i}\right|^{2}>1$ then $P(\mathbb{D}) \cap(\mathbb{C} \backslash \overline{\mathbb{D}}) \neq \emptyset$.

Proof That $\sup _{|z| \leq 1}|P(z)| \leq \sum_{i=0}^{n}\left|p_{i}\right|$ is clear. To obtain the other inequality apply Parseval's Formula to $P\left(e^{i \theta}\right)$ with $\theta \in[-\pi, \pi]$, and the Maximum Modulus Principle to get

$$
\sum_{i=0}^{n}\left|p_{i}\right|^{2}=\frac{1}{2 \pi} \int_{-\pi}^{\pi}\left|P\left(e^{i \theta}\right)\right|^{2} d \theta \leq\left(\sup _{|z| \leq 1}|P(z)|\right)^{2}
$$

If $1<\sum_{i=0}^{n}\left|p_{i}\right|^{2}$, we have that $P(\overline{\mathbb{D}}) \cap(\mathbb{C} \backslash \overline{\mathbb{D}}) \neq \emptyset$ since $\sup _{|z| \leq 1}|P(z)|$ is in fact a maximum reached at the boundary of the unit disc. The fact that $P(\mathbb{D})$ is open gives the conclusion.

Remark 1 We would like to point out that Lemma 1 is also valid, with the same proof, for holomorphic functions $f(z)=\sum_{k=0}^{\infty} \hat{f}_{k} z^{k}$ with $\|f\|_{1}=\sum_{k=0}^{\infty}\left|\hat{f}_{k}\right|<\infty$.

Remark 2 The chain of inequalities in Lemma 1 needs some clarifications. It is clear that if $P(z)$ is a monomial then nothing can be done since we have all equalities. If $P(z)$ is not a monomial, then one has $\left(\sum_{i=0}^{n}\left|p_{i}\right|^{2}\right)^{1 / 2}<\sum_{i=0}^{n}\left|p_{i}\right|$ and it is easy to find polynomials satisfying $\sup _{|z| \leq 1}|P(z)|=\sum_{i=0}^{n}\left|p_{i}\right|$ (take for example all coefficients to be positive real numbers). On the other hand, it is not clear at a first glance, if the inequality $\left(\sum_{i=0}^{n}\left|p_{i}\right|^{2}\right)^{1 / 2} \leq \sup _{|z| \leq 1}|P(z)|$ is optimal in the sense that for any $q<2$ arbitrarily close to 2 there exists $P(z)$ with $\sup _{|z| \leq 1}|P(z)| \leq\left(\sum_{i=0}^{n}\left|p_{i}\right|^{q}\right)^{1 / q}$. This is in fact the case as the following construction shows (see [12, p. 75]). We thank A. Peris for pointing out this to us.

Take $P_{0}(z):=z$ and $P_{j+1}(z):=P_{j}\left(z^{2}\right)+z^{-1} P_{j}\left(-z^{2}\right)$ for $j \geq 0$. It is easy to show that $P_{j}(z)=\sum_{k=1}^{2^{j}} p_{k} z^{k}$, where $p_{k}= \pm 1$ and thus $\left(\sum_{i=0}^{2^{j}}\left|p_{i}\right|^{q}\right)^{1 / q}=2^{j / q}$. By induction one may prove that if $|z|=1$ then $\left|P_{j}(z)\right|^{2}+\left|P_{j}(-z)\right|^{2}=2^{j+1}$. If we choose a positive integer $j>q /(2-q)$ we obtain

$$
\sup _{|z| \leq 1}|P(z)| \leq 2^{(j+1) / 2}<2^{j / q}=\left(\sum_{i=0}^{2^{j}}\left|p_{i}\right|^{q}\right)^{1 / q} .
$$

The next result is a complex variable exercise that will be used in Lemma 3. It provides the Taylor expansion of $g(z):=1 / f(z)=\sum_{m=0}^{\infty} g_{m}(z-a)^{m}$ in terms of the Taylor expansion of $f(z)=\sum_{m=0}^{\infty} f_{m}(z-a)^{m}$, provided $f(z)$ is analytic at $a$ and $f(a) \neq 0$. The result is probably known and its proof is included here for completeness since we were unable to find a reference for it. We need some matrix 
notation; for an analytic function $f(z)$ at $a$, write

$$
T_{f(a)}^{(0)}:=(1), \text { and } T_{f(a)}^{(m)}:=\left(\begin{array}{ccccccc}
\frac{f^{\prime}(a)}{1 !} & \frac{f^{\prime \prime}(a)}{2 !} & \frac{f^{\prime \prime \prime}(a)}{3 !} & & & \frac{f^{(m)}(a)}{m !} \\
f(a) & \frac{f^{\prime}(a)}{1 !} & \frac{f^{\prime \prime}(a)}{2 !} & \ddots & & \\
0 & f(a) & \frac{f^{\prime}(a)}{1 !} & \ddots & \ddots & \\
& \ddots & \ddots & \ddots & \ddots & \frac{f^{\prime \prime \prime}(a)}{3 !} \\
& & \ddots & \ddots & \ddots & \frac{f^{\prime \prime}(a)}{2 !} \\
0 & & & 0 & f(a) & \frac{f^{\prime}(a)}{1 !}
\end{array}\right) \text { for } m \in \mathbb{N} \text {. }
$$

In particular, for $m=1$ and $m=2$ we have

$$
T_{f(a)}^{(1)}=\left(f^{\prime}(a)\right), T_{f(a)}^{(2)}=\left(\begin{array}{cc}
f^{\prime}(a) & \frac{f^{\prime \prime}(a)}{2} \\
f(a) & f^{\prime}(a)
\end{array}\right) .
$$

Lemma 2 Let $f(z)$ be an analytic function at $a \in \mathbb{C}$. If $f(a) \neq 0$ then

$$
\left(\frac{1}{f}\right)^{(m)}(a)=(-1)^{m} \frac{m !}{f(a)^{m+1}} \operatorname{det} T_{f(a)}^{(m)}
$$

for each $m \in \mathbb{N}_{0}$.

Proof Write $g(z):=1 / f(z)$ and, without loss of generality, suppose $a=0$. Since $f(z)$ and $g(z)$ are analytic at 0 we have $f(z)=\sum_{m=0}^{\infty} f_{m} z^{m}$ and $g(z)=\sum_{m=0}^{\infty} g_{m} z^{m}$, where $f_{m}=f^{(m)}(0) / m$ ! and $g_{m}=g^{(m)}(0) / m$ ! respectively. Consider its Cauchy product to get

$$
\sum_{m=0}^{\infty}\left(\sum_{i+j=m} f_{i} g_{j}\right) z^{m}=1 .
$$

Clearly, $g_{0}=1 / f_{0}$ and $g_{1}=-f_{1} / f_{0}^{2}$. Suppose by induction that

$$
g_{k}=(-1)^{k} \frac{1}{f_{0}^{k+1}} \operatorname{det} T_{f(0)}^{(k)} \text { for } k<m .
$$

Since $\sum_{i+j=m} f_{i} g_{j}=0$, we have

$$
\begin{aligned}
g_{m} & =-\sum_{i=0}^{m-1} f_{i} g_{m-i} / f_{0} \\
& =\frac{-1}{f_{0}}\left(\frac{f_{m}}{f_{0}}+\sum_{i=1}^{m-1} \frac{(-1)^{m-i} f_{i}}{f_{0}^{m-i+1}} \operatorname{det} T_{f(0)}^{(m-i)}\right) \\
& =\frac{-1}{f_{0}^{m+1}}\left(f_{m} f_{0}^{m-1}+\sum_{i=1}^{m-1}(-1)^{m-i} f_{i} f_{0}^{i-1} \operatorname{det} T_{f(0)}^{(m-i)}\right) \\
& =\frac{(-1)^{m}}{f_{0}^{m+1}}\left(\sum_{i=1}^{m-1}(-1)^{1+i} f_{i} f_{0}^{i-1} \operatorname{det} T_{f(0)}^{(m-i)}+(-1)^{1+m} f_{m} f_{0}^{m-1}\right) \\
& =\frac{(-1)^{m}}{f_{0}^{m+1}} \operatorname{det} T_{f(0)}^{(m)},
\end{aligned}
$$

as desired. 
Lemma 3 Let $P(z)=p_{0}+p_{1} z+\ldots+p_{n} z^{n}$ be a complex polynomial.

(i) If $\left|p_{0}\right|-\left|p_{n}\right|<1$, then $P(\mathbb{D}) \cap \mathbb{D} \neq \emptyset$

(ii) $\left|P(a)^{m+1}\right|<(1-|a|)^{m}\left|\operatorname{det} T_{P(a)}^{(m)}\right|$ for some $a \in \mathbb{D}$ and some $m \in \mathbb{N}_{0}$ if, and only if, $P(\mathbb{D}) \cap \mathbb{D} \neq \emptyset$.

Proof (i) Assume $p_{0} \neq 0$. First we shall see that there exists $b \in \mathbb{D}$ such that $\left|p_{0}-b\right|<\left|p_{n}\right|$. For that fix $0 \leq r<\min \left\{1,\left|p_{0}\right|\right\}$ such that $\left|p_{0}\right|-\left|p_{n}\right|<r$ and take $b:=\left(r /\left|p_{0}\right|\right) p_{0}$. We have $\left|p_{0}-b\right|=|| p_{0}|-r|<\left|p_{n}\right|$. Now consider the polynomial $Q(z):=P(z)-b$; its factorization provides $Q(z)=p_{n}\left(z-\alpha_{1}\right) \cdots\left(z-\alpha_{n}\right)$. Easy computations show that

$$
p_{n}(-1)^{n} \prod_{k=1}^{n} \alpha_{k}=Q(0)=p_{0}-b,
$$

and then

$$
\prod_{k=1}^{n}\left|\alpha_{k}\right|=\frac{\left|p_{0}-b\right|}{\left|p_{n}\right|}<1 .
$$

This ensures the existence of $k_{0}$ such that $\left|\alpha_{k_{0}}\right|<1$ and, since $Q\left(\alpha_{k_{0}}\right)=0$, we have $P\left(\alpha_{k_{0}}\right)=b$.

(ii) If there exists $a \in \mathbb{D}$ such that $P(a) \in \mathbb{D}$, then the inequality holds for such $a$ and $m=0$. For the converse we proceed by contradiction. If we suppose $|P(z)| \geq 1$ for all $z \in \mathbb{D}$ then the function $g(z):=1 / P(z)$ is holomorphic in $\mathbb{D}$ with $|g(z)| \leq 1$ for all $z \in \mathbb{D}$. Cauchy's Estimates give $\left|g^{(m)}(a)\right| \leq \frac{m !}{(1-|a|)^{m}}$ for each $a \in \mathbb{D}$ and each $m \in \mathbb{N}_{0}$. Using Lemma 2, we get $\left|\operatorname{det} T_{P(a)}^{(m)}\right| \leq \frac{\left|P(a)^{m+1}\right|}{(1-|a|)^{m}}$ for each $a \in \mathbb{D}$ and each $m \in \mathbb{N}_{0}$.

Remark 3 It is tempting to think that $|P(a)|-\left|p_{n}\right|<1$ for some $a \in \mathbb{D}$ implies that $P(\mathbb{D}) \cap \mathbb{D} \neq \emptyset$ but this does not hold. For instance, for $2<q$ and $P(z)=3 q / 2+q z$ we have $|P(-1 / 2)|-q=0$ but $|P(z)|>1$ for all $z \in \mathbb{D}$.

Theorem 1 Let $1 \leq p<\infty$ and $P(z)=p_{0}+p_{1} z+\ldots+p_{n} z^{n}$ be a complex polynomial with $\sum_{k=0}^{n}\left|p_{k}\right|^{2}>1$.

(i) If $\left|p_{0}\right|-\left|p_{n}\right|<1$ then $p(B)$ is chaotic on $\ell^{p}$.

(ii) $P(B)$ is chaotic on $\ell^{p}$ if, and only if, $\left|P(a)^{m+1}\right|<(1-|a|)^{m}\left|\operatorname{det} T_{P(a)}^{(m)}\right|$ for some $a \in \mathbb{D}$ and some $m \in \mathbb{N}_{0}$.

In particular, if

$$
\left|p_{0}^{m+1}\right|<\operatorname{det}\left(\begin{array}{cccccc}
p_{1} & p_{2} & p_{3} & & & p_{m} \\
p_{0} & p_{1} & p_{2} & \ddots & & \\
0 & p_{0} & p_{1} & \ddots & \ddots & \\
& \ddots & \ddots & \ddots & \ddots & p_{3} \\
& & \ddots & \ddots & \ddots & p_{2} \\
0 & & & 0 & p_{0} & p_{1}
\end{array}\right) \mid,
$$

for some $m \in \mathbb{N}$ (we set $p_{i}=0$ for $i>n$ ), then $P(B)$ is chaotic on $l^{p}$. 
Proof Apply Lemma 1, Lemma 3, and the characterization in [6, Theorem 2.3]. Observe that Theorem 2.3 in [6] has a required condition named (H1), this condition holds in our case.

Next we will study some special cases and examples. First observe that to get chaos of $P(B)$ we must assume $\sum_{i=0}^{n}\left|p_{i}\right|>1$ because $\|P(B)\| \leq \sum_{i=0}^{n}\left|p_{i}\right|$ and operators with norm less than or equal to 1 cannot be hypercyclic.

Example 1 (1) If $P(z)=p_{n} z^{n}$ with $n>0$, we have that $P(B)$ is chaotic if and only if $\left|p_{n}\right|>1$.

(2) If $P(z)=p_{0}+p_{n} z^{n}$ with $p_{n} \neq 0$, we have that $P(B)$ is chaotic if and only if $|1-| p_{0}||<\left|p_{n}\right|$. This generalizes affine functions of $B$ (see [6, Corollary 2.11] and $[16])$.

(3) If $P(z)=p_{m} z^{m}+p_{n} z^{n}$ with $0<m<n$, then $P(B)$ is chaotic if and only if $\left|p_{m}\right|+\left|p_{n}\right|>1$. To obtain this characterization you have to apply Theorem 1 , the comment previous to these examples and that $\sup _{|z| \leq 1}|P(z)|=\left|p_{m}\right|+\left|p_{n}\right|$ since $\left|P\left(e^{i \theta}\right)\right|=\left|p_{m}\right|+\left|p_{n}\right|$ for $\theta:=\left(\alpha_{m}-\alpha_{n}\right) /(n-m)$, where $p_{m}=\left|p_{m}\right| e^{i \alpha_{m}}$ and $p_{n}=\left|p_{n}\right| e^{i \alpha_{n}}$.

(4) If $\left|p_{0}\right|<1+\left|p_{n}\right|$ and $\sum_{k=0}^{n}\left|p_{k}\right|^{2}>1$ then $P(B)$ is chaotic.

(5) The two previous cases raise the following natural question: If $p_{0}=0$ and $\sum_{k=1}^{n}\left|p_{k}\right|>1$, must $P(B)$ be chaotic? The answer is not as the following example shows. Take $P(z)=z / 3+z^{2} / 3-z^{3} / 2$. In this case $P(\mathbb{D}) \subset \mathbb{D}$ since $\sup _{|z| \leq 1}|P(z)|=|P(\exp (i \arccos (-1 / 12)))|<1$.

Next, we want to study chaos for $P(B)$ defined on weighted $\ell^{p}(v)$ spaces. It is easy to see that $\varrho \mathbb{D} \subset \sigma_{p}(B)$, where $\varrho^{p}=\liminf _{i} v_{i}{ }^{-1 / i}$. Unfortunately, in this framework, chaos of $P(B)$ is not equivalent to $P(\varrho \mathbb{D}) \cap \mathbb{T} \neq \emptyset$. Take $P(z)=z$ and the spaces $\ell^{1}, \ell^{1}(1 / n)$, and $\ell^{1}\left(1 / n^{2}\right)$. In all three cases $\varrho=1$ and $P(\mathbb{D}) \cap \mathbb{T}=\emptyset$; however $B$ is not hypercyclic on $\ell^{1}$, it is hypercyclic but not chaotic on $\ell^{1}(1 / n)$, and it is chaotic on $\ell^{1}\left(1 / n^{2}\right)$. For this reason we will use only that $P(B)$ is chaotic on $\ell^{p}(v)$ if $P(\varrho \mathbb{D}) \cap \mathbb{T} \neq \emptyset$.

Before we state the theorem providing chaos for $P(B)$ on $\ell^{p}(v)$, let us remark that $\varrho<\infty$. Indeed, the weight condition $\left(^{*}\right)$ implies that there exists $M>0$ such that for each $i \geq 2$ we have $v_{i}>v_{i-1} / M$. Inductively $v_{i}>v_{1} M^{1-i}$ for all $i>1$ and therefore $\varrho^{p} \leq M<\infty$. Also, we disregard $\varrho=0$ since this case forces $B$ to be a compact operator as so is any $B^{n}$ for each $n \geq 1 ; P(B)$ has a chance to be hypercyclic only if $\left|p_{0}\right|=1$ but compact perturbations of $p_{0} I$ are never chaotic (see [13, Proposition 6.1]).

Theorem 2 Let $P(z)=p_{0}+p_{1} z+\cdots+p_{n} z^{n}$ be a complex polynomial and let $v=$ $\left(v_{i}\right)_{i}$ be a sequence satisfying the weight condition $\left(^{*}\right)$ such that $\sum_{k=0}^{n}\left|\varrho^{k} p_{k}\right|^{2}>1$, where $\varrho^{p}=\liminf _{i} v_{i}^{-1 / i}>0$. 
If $\left|p_{0}\right|-\left|\varrho^{n} p_{n}\right|<1$ or $\left|P(\varrho a)^{m+1}\right|<(1-|a|)^{m}\left|\operatorname{det} T_{P(\varrho a)}^{(m)}\right|$ for some $a \in \mathbb{D}$ and $m \in \mathbb{N}$, then $P(B)$ is chaotic on $\ell^{p}(v)$. In particular, if

$$
\left|p_{0}^{m+1}\right|<\operatorname{det}\left(\begin{array}{cccccc}
\varrho p_{1} & \varrho^{2} p_{2} & \varrho^{3} p_{3} & & & \varrho^{m} p_{m} \\
p_{0} & \varrho p_{1} & \varrho^{2} p_{2} & \ddots & & \\
0 & p_{0} & \varrho p_{1} & \ddots & \ddots & \\
& \ddots & \ddots & \ddots & \ddots & \varrho^{3} p_{3} \\
& & \ddots & \ddots & \ddots & \varrho^{2} p_{2} \\
0 & & & 0 & p_{0} & \varrho p_{1}
\end{array}\right) \mid,
$$

for some $m \in \mathbb{N}$ (we set $p_{i}=0$ for $\left.i>n\right)$, then $P(B)$ is chaotic on $\ell^{p}(v)$.

Proof Clearly $P(\varrho \mathbb{D}) \cap \mathbb{T} \neq \emptyset$ if and only if $Q(\mathbb{D}) \cap \mathbb{T} \neq \emptyset$, where $Q(z)=P(\varrho z)$.

Remark 4 Some operators can be represented as a weighted backward shift operator $B_{w}\left(x_{1}, x_{2}, \ldots\right):=\left(w_{2} x_{2}, w_{3} x_{3}, \ldots\right)$ defined on a weighted $\ell^{p}(v)$ space. This case may be reduced to the previous one via topological conjugacy. Set

$$
a_{1}:=1, a_{i}:=w_{2} \ldots w_{i}, i>1,
$$

and consider $\ell^{p}(\bar{v})$ where

$$
\bar{v}_{i}=\frac{v_{i}}{\prod_{j=2}^{i} w_{j}^{p}} \text {, for all } i .
$$

Take $\phi_{a}: \ell^{p}(v) \rightarrow \ell^{p}(\bar{v})$ defined as $\phi_{a}\left(x_{1}, x_{2}, \ldots\right):=\left(a_{1} x_{1}, a_{2} x_{2}, \ldots\right)$ to construct a commutative diagram $\phi_{a} \circ B_{w}=B \circ \phi_{a}$. Since $\phi_{a}$ is an isometry, by topological conjugacy, we have that $P(B)$ is chaotic on $\ell^{p}(\bar{v})$ if and only if $P\left(B_{w}\right)$ is chaotic on $\ell^{p}(v)$.

Differential operators on Hilbert spaces of entire functions (see [4]). Let $\gamma(z)$ be an admissible comparison entire function, that is, the Taylor coefficients $\gamma_{i}>0$ for all $i \in \mathbb{N}_{0}$ and the sequence $\left(i \gamma_{i} / \gamma_{i-1}\right)_{i \geq 1}$ is monotonically decreasing. We consider the Hilbert space $E^{2}(\gamma)$ of power series

$$
g(z)=\sum_{i=0}^{\infty} \hat{g}(i) z^{i}
$$

for which

$$
\|g\|_{2, \gamma}^{2}:=\sum_{i=0}^{\infty} \gamma_{i}^{-2}|\hat{g}(i)|^{2}<\infty .
$$

Chan and Shapiro showed that for $a \neq 0$ the translation operator $T_{a}$ is hypercyclic on $E^{2}(\gamma)$ (see [4, Theorem 2.1]. They proved that $T_{a}=\sum_{n>0} a^{n} / n ! D^{n}$, where $D$ is the operator of differentiation. They pointed out that the hypercyclicity of $T_{a}$ is in fact the hypercyclicity of $f(D)$ for the particular case $f(z)=e^{a z}$. They also asked for the dynamics of 'other' operators; we will consider here differential operators $P(D)$. 
It is clear that $E^{2}(\gamma)$ is isometric to $\ell^{2}(v)$ with $v=\left(v_{i}\right)_{i \in \mathbb{N}_{0}}=\left(\gamma_{i}^{-2}\right)_{i \in \mathbb{N}_{0}}$ and with the identification $f \mapsto\left(f^{(i)}(0) / i \text { ! }\right)_{i \geq 0}$. Moreover, the operator of differentiation $D$ turns out to be a weighted backward shift with weights $w=\left(w_{i}\right)_{i \geq 1}=$ $(i)_{i \geq 1}$ or, equivalently, as a backward shift defined on $\ell^{2}(\bar{v})$, where

$$
\bar{v}_{i}=\frac{1}{\left(\gamma_{i} i !\right)^{2}}, i \geq 0
$$

Since $\gamma(z)$ is an admissible comparison entire function, it is easy to check that the weight condition $\left(^{*}\right)$ is satisfied and $P(B)$ is a bounded operator on $\ell^{2}(\bar{v})$ for any polynomial $P(z)$ (this is equivalent to saying that $P(D)$ is a bounded operator on $E^{2}(\gamma)$ for any polynomial $\left.P(z)\right)$.

Let us now consider only those spaces for which $\lim _{i \rightarrow \infty} i \gamma_{i} / \gamma_{i-1}>0$. Since the limit of successive roots coincides with the limit of successive ratios we have that $\varrho=\lim _{i \rightarrow \infty} i \gamma_{i} / \gamma_{i-1}>0$, and Theorem 2 may be applied to polynomials $P(z)$.

Example 2 To focus on a concrete example take $\gamma(z)=e^{\alpha z}$ with $\alpha>0$; it is clear that $\varrho=\alpha$. Considering chaos for differential operators $P(D)$ on $E^{2}\left(e^{\alpha z}\right)$, we have that

(1) $a D^{n}$ with $n>0$ is chaotic if and only if $\alpha^{n}|a|>1$.

(2) $a+b D^{n}$ with $b \neq 0$ is chaotic if and only if $|1-| a||<\left|\alpha^{n} b\right|$.

(3) $a D^{n}+b D^{m}$ with $0<m<n$ is chaotic if and only if $\alpha^{n}|a|+\alpha^{m}|b|>1$.

(4) $P(D)=\sum_{k=0}^{n} p_{k} D^{k}$ is chaotic if $\left|p_{0}\right|<1+\alpha^{n}\left|p_{n}\right|$ and $\sum_{k=0}^{n} \alpha^{k}\left|p_{k}\right|^{2}>1$.

Backward shift operators on spaces of analytic functions. Although one of our initial motivations to start this research was to study chaos for differential operators on Hilbert spaces of entire functions, our results may also be applied to other classical spaces of analytic functions. Consider spaces consisting on formal power series $f(z):=\sum_{i=0}^{\infty} \hat{f}_{i} z^{i}$ for which the sequence $\left(\hat{f}_{i}\right)_{i}$ belongs to $\ell^{2}(v)$ for a certain weight sequence $v=\left(v_{i}\right)_{i}$. For example, the Hardy space $H^{2}$ is obtained for $v=(1)_{i}$, the Bergman space $A^{2}$ for $v=(1 /(1+i))_{i}$, and the Dirichlet space $\mathcal{D}$ for $v=(i+1)_{i}$. For the orthogonal basis $\left(z^{i}\right)_{i>0}$, the backward shift is defined as $B\left(z^{i}\right):=z^{i-1}$ for $i \in \mathbb{N}$, and $B(1)=0$. Now, if $P(z)$ is a non-constant polynomial, since $\varrho^{2}=\liminf _{i} v_{i}^{-1 / i}=1$ for the spaces $H^{2}, A^{2}$, and $\mathcal{D}$, chaos of $P(B)$ may be obtained using Theorem 1.

Future work and open problems. In Theorem 1 we give sufficient conditions on the coefficients of the polynomial $P(z)$ in order to have chaos for the operator $P(B)$ defined on $\ell^{p}$. As one may expect, polynomials are not the end of the story; for functions $f(z)$ holomorphic in $\alpha \mathbb{D}$ for some $\alpha>1$, the usual Riesz functional calculus defines a bounded operator $f(B)$ on $\ell^{p}$. It is known that for such (nonconstant) holomorphic functions, the operator $f(B)$ is chaotic on $\ell^{p}$ if and only if $f(\mathbb{D}) \cap \mathbb{T} \neq \emptyset$ (see [6, Theorem 2.3]). Since a full geometrical description of $f(\mathbb{D})$ may be hard to find in general it would be nice to have some 'computable' conditions for chaos of $f(B)$, at least for some particular classes of functions. For example, if $f(z)=a /(z-b)$ where $a$ and $b$ are complex numbers with $|b|>1$, since $\sigma(B)=\overline{\mathbb{D}}$, we have that $f(B)=a(B-b)^{-1}$ is a bounded operator on $\ell^{p}$. It can be checked that $f(\mathbb{D})$ is the open disc $c+r \mathbb{D}$ where

$$
c=-\frac{a}{b} \frac{|b|^{2}}{|b|^{2}-1} \text { and } r=\frac{|a|}{|b|^{2}-1},
$$


hence $f(B)$ is chaotic if and only if ||$b|-| a||<1$.

The meromorphic case is a more general and difficult problem: Suppose $f(z)=$ $P(z) / Q(z)$ where $P(z)$ and $Q(z)$ are complex polynomials with all roots of $Q(z)$ lying outside the closed unit disk. As before $f(B)$ is a bounded operator on $\ell^{p}$. What conditions on the coefficients (or on the roots) of $P$ and $Q$ ensure chaos for $f(B)$ ?

Acknowledgements This work was partially supported by the MEC and FEDER Projects MTM2007-64222, MTM2010-14909, and by GVA Project GV/2010/091, and by UPV Project PAID-06-09-2932. The authors would like to thank A. Peris for helpful comments and ideas that produced a great improvement of the paper's presentation. We also thank the referees for their helpful comments and for reporting to us a gap in Theorem 1.

\section{References}

1. Frédéric Bayart and Étienne Matheron, Dynamics of linear operators, Cambridge Tracts in Mathematics, vol. 179, Cambridge University Press, Cambridge, 2009.

2. Teresa Bermúdez and Vivien G. Miller, On operators $T$ such that $f(T)$ is hypercyclic, Integral Equations Operator Theory 37 (2000), no. 3, 332-340.

3. J. Bonet, F. Martínez-Giménez, and A. Peris, Linear chaos on Fréchet spaces, Internat. J. Bifur. Chaos Appl. Sci. Engrg. 13 (2003), no. 7, 1649-1655.

4. Kit C. Chan and Joel H. Shapiro, The cyclic behavior of translation operators on Hilbert spaces of entire functions, Indiana Univ. Math. J. 40 (1991), no. 4, 1421-1449.

5. José A. Conejero and Vladimír Müller, On the universality of multipliers on $\mathcal{H}(\mathbb{C})$, J. Approx. Theory 162 (2010), no. 5, 1025-1032.

6. R. deLaubenfels and H. Emamirad, Chaos for functions of discrete and continuous weighted shift operators, Ergodic Theory Dynam. Systems 21 (2001), no. 5, 1411-1427.

7. Robert L. Devaney, An introduction to chaotic dynamical systems, second ed., AddisonWesley Studies in Nonlinearity, Addison-Wesley Publishing Company Advanced Book Program, Redwood City, CA, 1989.

8. Gilles Godefroy and Joel H. Shapiro, Operators with dense, invariant, cyclic vector manifolds, J. Funct. Anal. 98 (1991), no. 2, 229-269.

9. K.-G. Grosse-Erdmann, Hypercyclic and chaotic weighted shifts, Studia Math. 139 (2000), no. $1,47-68$.

10. K.-G. Grosse-Erdmann and A. Peris, Linear chaos, Universitext, Springer, New York, to appear in 2011.

11. Gerd Herzog and Christoph Schmoeger, On operators $T$ such that $f(T)$ is hypercyclic, Studia Math. 108 (1994), no. 3, 209-216.

12. Jean-Pierre Kahane, Some random series of functions, second ed., Cambridge Studies in Advanced Mathematics, vol. 5, Cambridge University Press, Cambridge, 1985.

13. F. Martínez-Giménez and A. Peris, Chaos for backward shift operators, Internat. J. Bifur. Chaos Appl. Sci. Engrg. 12 (2002), no. 8, 1703-1715.

14. Félix Martínez-Giménez, Chaos for power series of backward shift operators, Proc. Amer. Math. Soc. 135 (2007), 1741-1752.

15. Vladimír Müller, On the Salas theorem and hypercyclicity of $f(T)$, Integral Equations Operator Theory 67 (2010), no. 3, 439-448.

16. V. Protopopescu and Y. Y. Azmy, Topological chaos for a class of linear models, Math. Models Methods Appl. Sci. 2 (1992), no. 1, 79-90.

17. S. Rolewicz, On orbits of elements, Studia Math. 32 (1969), 17-22.

18. Héctor N. Salas, Hypercyclic weighted shifts, Trans. Amer. Math. Soc. 347 (1995), no. 3, 993-1004.

19. Joel H. Shapiro, Simple connectivity and linear chaos, Rend. Circ. Mat. Palermo (2) Suppl. (1998), no. 56, 27-48. 